\title{
Efficient resource allocation and dimensioning of media edge clouds infrastructure
}

\author{
Abdallah Jarray*, Ahmed Karmouch, Javier Salazar, Jocelyne Elias, Ahmed Mehaoua and Faisal Zaman
}

\begin{abstract}
Media Edge Cloud Data Centers (MEC-DCs) that are interconnected by a Metro Network were selected as infrastructure to enhance the Quality of Experience (QoE) for end users of multimedia applications. Unlike the traditional Data Centers, MEC-DCs, which are kept closer to the user, have limited availability of resources at a given Data Center. Therefore, it is of paramount importance for Infrastructure service providers to efficiently dimension and use the media resources in an environment where the applications have high resource demand and the infrastructure has limited availability. To perform this task dynamically, we first propose a resource allocation strategy that considers the physical characteristics of the networking layer while minimizing the costs of deploying media applications. Second, we analyze the different configurations of the networking layer in order to enhance the use of MEC-DCs resources and the QoE for the end-users. Simulation results show a clear advantage of this proposed optimization-based approach over the benchmarks in terms of provisioning costs, blocking ratio and resource use.
\end{abstract}

Keywords: Media cloud service, Media edge data center, Resource allocation, Cloud provider, Optical network, Linear programming, Column generation

\section{Introduction}

The evolution in network technologies is changing the way in which communications are designed. With the development of Web 2.0 that supports multimedia applications, customer expectations of rich media provisioning have increased. Media applications are becoming essential to our everyday life [1]; their popularity is increasing both because of the spread of social networks and the ease with which they can share audio, videos, and streaming services.

Media applications can vary from video sharing (such as YouTube and Netflix), to online radio (Spotify) or image sharing (Pinterest). Most of these services demand a significant amount of media processing and have stringent Quality of Service (QoS) requirements [2]. This is particularly the case with User Generated Content (UGC), with its huge volume of short videos and its significantly fluctuating user demand. Cloud computing is

*Correspondence: ajarray@uottawa.ca

University of Ottawa, School of Electrical Engineering and Computer Science, Ottawa, Ontario, Canada gaining enormous momentum as a cost-efficient solution for providing media services with storage and processing requirements [3]. Large-scale public clouds that offer their computing, storage, and network resources in the form of Infrastructure-as-a-Service (IaaS) have attracted Cloud Service Providers (CPs) [4-6].

A current area of rapid innovation is the use of Media Edge Cloud Data Centers (MEC-DCs) using several hundred servers $[7,8]$. This MEC model allows CPs to reduce their capital costs and to benefit from the elasticity of the cloud by placing Virtual Machines (VM) running media processing tasks closer to the end-users.

The Edge Cloud infrastructure uses smaller DCs located in the last mile, closer to major population centers, in order to honor Service Level Agreement (SLA) contracts for the QoS requirements of highly interactive content delivery: online searching (such as Google), social networking (such as Facebook), video streaming, and so on [8]. This requires a topology located in the metropolitan area that can transfer media data among DC locations. Metro optical fiber networks have been investigated as the best way to guarantee an efficient data transport 
service among MEC-DC locations [9]. Metro optical networks can indeed transport large files with short time delays, making for a suitable architecture for applications where avoiding delay is critical, such as on-line gaming, video streaming and image sharing. Advancements in optical communication have enabled the grooming of low-granularity traffic using optical Multi-service Provisioning Platform (MSPP) transponders [10]. Examples are the media applications in social networks, often in the form of small files like profile pictures or short videos.

To ensure the SLA of the hosted applications, it is important that the cloud substrate resources and the link latency constraints are satisfied. The presence of MECDCs closer to the user gives service providers two advantages over traditional DCs, (a) a reduction in the cost/bit, and $(b)$ an increased performance and throughput of the application. On the other hand, compared to traditional DC networks, MEC-DCs have limited resources at a given site. Smart site selection is important in order to ensure both QoS and a minimum cost for the CPs. It is our belief that overall substrate resource use is improved if the incoming media cloud request is mapped to the MEC-DCs simultaneously for both its networking and computing requirements. Our approach, proposed in "Media edge cloud resource allocation approach" section, has two crucial improvements over related works in the literature [11-20,20]. The first improvement is a resource allocation strategy that uses the physical characteristics of the networking layer to reduce the deployment cost. The strategy, referred to as L-CG-MEC, uses Column Generation as a large-scale optimization technique for mapping media cloud requests to the MEC-DCs. L-CGMEC defines a media cloud request as a Virtual Network (VN); a set of nodes and a set of links with QoS requirements. The second improvement is to evaluate different networking configurations so as to determine which can provide better QoS.

The remainder of this paper is organized as follows. "Related works" section describes other work related to our proposal. "Media cloud computing" section defines the MEC-DCs resources allocation problem. "Media edge cloud resource allocation approach" section presents the mapping solution of media cloud requests into MEC-DCs infrastructure. "Numerical results" section introduces benchmarks and simulation results to evaluate performance. "Conclusion" section concludes the paper.

\section{Related works}

The literature contains a number of approaches to efficiently solving the challenges of media cloud requests mapping. This can be defined as mapping a set of incoming media cloud requests on the MEC-DCs infrastructure so as to enhance goals such as reducing cost, increasing profit, or network use. It is important to ensure that the
QoS constraints of the incoming requests are satisfied. The challenges mainly result from the increased computational complexity when media cloud computing and the requirements of networking resources are considered jointly.

To overcome these issues, proposals in the literature have considered either relaxing networking QoS by focusing only on the computing requirements $[11,13]$ or adopting a two-phase approach $[14,15,18]$, which first pre-selects the mapping of hosting nodes and then maps virtual links.

The authors in [11] present a Bin-packing approach that dynamically maps Virtual Machines (VMs) into Physical Machines (PMs). As a result, networking requirements are not considered in the optimization model, which may mean that QoS requirements are not met.

In [12], the authors introduce an optimization algorithm based on a multi-objective formulation that optimizes the power used as well as the load balancing among DC servers. But the cost of networking equipment is not considered. The model therefore lacks a realistic evaluation of the economic benefits of cloud service requests and could also result in QoS requirements not being met.

In [13], the proposal is for two cloud VN embedding approaches using an optical network. The author's focus is to minimize the power and spectrum used. The proposal does not detail the dimensioning of the optical layer and the impact of networking parameters on the quality of the services offered. The parameters of the optical networking layer are likewise not considered.

In [14], the authors use a two-phase mapping approach, which first preselects the mapping of hosting nodes and then maps virtual links. Node mapping and link mapping are performed independently. Hence, non-join node and link embedding may result in a high number of blocked requests and in underused resources, meaning less profit for the cloud provider. In addition, the mapping is done using heuristic approaches, which may make the solution less than optimal.

The authors in [15] propose a mathematical programming scheme in order to coordinate node and link mapping. The proposal handles online Virtual Network requests and introduces a better correlation between virtual node and virtual link embedding phases. However, the solution seems less satisfactory than simultaneous mapping.

In [18], the authors propose a greedy algorithm that jointly optimizes the global workload assignment and the local VM allocation in order to minimize the resource cost under the response time requirements. While the focus is on the media cloud request and the stringent QoS requirements, the analysis does not study the impact of the key networking layer factors on performance. 
The authors of [19] study a joint optimization model that is geographically distributed and interconnected using an optical network. However, the resource allocation applies more to a general IaaS request model. The impact of the networking layer on a higher QoS received much less attention.

The proposal in [20] is possibly the closest to our work. The light-trial approach adapts well for multi-casting applications. The proposal also considers the characteristics of the optical layer. However, the authors do not analyze the impact of the networking layer on the QoS for media cloud applications and their resource allocation proposal is better suited to general IaaS requirements.

In [21], the authors propose a next-generation, ubiquitous, converged infrastructure. The proposal connects fixed and mobile end users with Data Centers through a heterogeneous, network-integrating optical metro network, based on time-shared network technology and wireless access. The approach ensures allocation of the required resources across all technology domains to support their specific characteristics such as end users' mobility.

In [22], the authors provide a VN planning scheme with the development of Wavelength Division Multiplexing (WDM) techniques and cloud computing. The approach uses a united virtualization of optical and server resources that collaboratively incorporates the optical backbone into Data Centers. The authors demonstrate the effectiveness of their strategy in the context of power outages and evolving recovery.

In [23], the authors use compressive sensing (CS) techniques to support scalable service provisioning in converged optical/wireless clouds. They claim that the CS techniques achieve optimal service provisioning with significantly reduced control and less computational complexity.

In [24], the authors use a column generation approach for the $\mathrm{VN}$ embedding. Their focus is on ensuring resiliency for the accepted $\mathrm{VN}$ requests. The network is formed by interconnected, geo-distributed DCs that are not limited by their computing resources. In this paper, the network topology is composed of Edge DCs with limited resources. As a result, the focus of our work is on ensuring QoS even with those limited resources.

In [25], the authors propose a VN embedding approach on a wireless optical network. Incoming $\mathrm{VN}$ requests are mapped to a local Wireless Mesh Network (WMN) so as to reduce the transmission power. If a request is not satisfied by the WMN, it is mapped to the Optical Edge Network. The main constraints, however, are the computational power and the wavelength availability. In addition, the modeling ignored several optical network attributes such as node grooming capacity and different optical architectures. In our work, we consider the optical network characteristics in order to make an informed decision on the VNs' mapping location and the availability of DC resource.

In [26], the authors propose an approach to determine the risk associated with a given Virtual Machine using threat and vulnerability factors. These factors identify which incoming $\mathrm{VN}$ requests can be risky. The main decision on the location of VM is governed by how risky the $\mathrm{VN}$ requests are. In our work, by contrast, the decision is based on the characteristics and performance of the network.

Most of these works had the following common features: (1) the use of two-phase VN mapping, (2) the general Infrastructure as a Service (IaaS) requirements, and (3) the mapping of $\mathrm{VN}$ request one at a time (i.e., online). Our proposal differs as follows: (1) for each accepted media cloud request, we calculate the optimal one-shot networking and hosting scheme with respect to QoS requirements (latency, bandwidth, computing, and mapping location). This guarantees a better use of MECDCs resources and an increased number of accepted VN requests, (2) media cloud requests are served by batch (see "Small-batch MEC mapping" section) which allows us to calculate a better mapping solution over time.

\section{Media cloud computing \\ Media edge cloud architecture}

Two classes of DC-based cloud architecture can be identified. They are (1) large, geographically distributed DCs, and (2) MEC-DCs as shown in Fig. 1. Large DCs are centralized and highly manageable, thereby providing an economy of scale. However, geographically distributed DCs have inherent limitations in service hosting. Simple economic factors determine that they are built only in locations where capital and operational costs are low. Large DCs are therefore generally located far from endusers. This may result in failed QoS requirements (such as latency and bandwidth/throughput) as well as higher networking costs. To address these drawbacks, Edge DCs (such as Micro-DCs and Edge Cloud) have been proposed. This new class of small-scale DCs, known as MEC-DCs, is well suited to service hosting. In MEC architecture, media content and processing are pushed to the edge of the cloud based on user profile.

\section{Networking transport architecture}

As mentioned, MEC-DC locations are interconnected using a metro optical network. A metro optical network is able to transport large files with short time latency, making it suitable for critical-delay applications such as on-line video gaming, video streaming and image sharing. An optical light-path transport architecture is used to allow grooming of low-granularity traffic using optical 


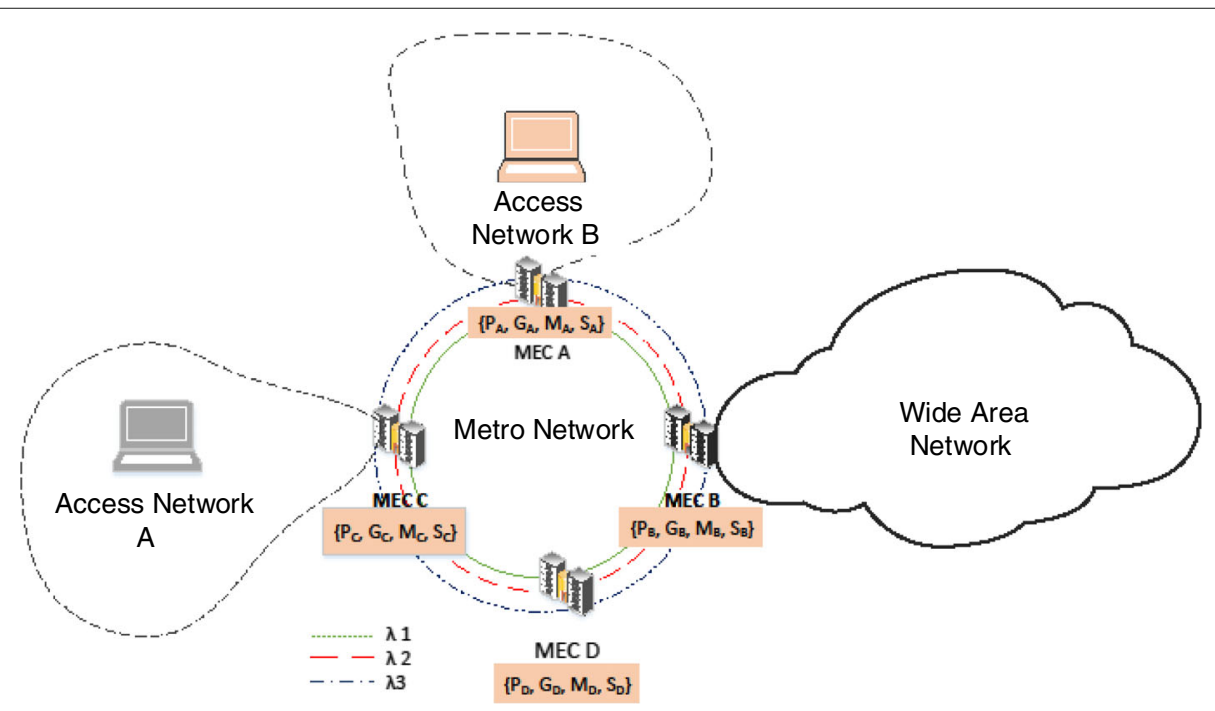

Fig. 1 Position of media edge clouds in Internet

MSPP transponders. We provide details in the following sections on the transport architecture.

\section{Light-Path transport architecture}

Data flows among MEC-DC locations are transported using a set of light-paths built on wavelengths available on each fiber link that interconnects MEC locations. A lightpath is an optical routing path that allows communication between the set of nodes along the path. A light-path uses only one wavelength between its end-nodes. A Multiservice Provisioning Access Platform (MSPP) transponder is used to add signals to or drop them from a wavelength at a given MEC-DC node.

\section{Add/Drop media request in edge cloud node}

A Multi-Service Provisioning Platform (MSPP) fabric is set up in each node of the interconnecting MEC-DC locations. MSPPs allow data flow to be added to or dropped from network transport signals according to traffic demand. Apart from the conventional SONET signals, MSPPs handle a wide variety of client signals (Gigabit Ethernet, ATM, IP, and so on). Furthermore, the MSPP equipment is modular and can be configured by selecting the component appropriate for the desired networking task for a given node [10]. Of these components, transponders, the interfaces between the optical and the electrical domains, make up the main element of cost on a MSPP fabric. MSPP transponders are used to add/drop and groom low-client signals into wavelengths.

\section{Media edge cloud resource allocation approach}

Resource allocation is one of the most important aspects of MEC-DCs management, since it is directly related to the cost and the QoS requirements of media cloud services. Efficient resource allocation has a positive impact on the service provider's profitability. The resource allocation problem is to minimize hosting and networking costs while preserving QoS constraints. The QoS requirements are: (1) Specific data transfer capacity with a short latency, (2) Pre-defined computing and graphic processing capacities, (3) Storage and memory capacity, and (4) Specific processing order of each task to compose the media cloud request. MEC-DCs Resources are allocated to the incoming media cloud requests in a batch-wise fashion, as described in the following section.

\section{Small-batch MEC mapping}

In a realistic scenario, media cloud requests usually do not arrive one after another at regular time intervals [15]. A realistic mapping scenario of $\mathrm{MEC}$ requests may therefore involve an approach, in which MEC requests are queued and then processed in small batches in order to optimize costs for the MEC provider over time [17]. To do so, we divide the mapping planning time into a set of consecutive short periods (windows) and we describe the demand with a set of MEC requests, one for each new window. From one period to the next, we assume that most MEC requests remain the same, representing as an example the global steady-state of the long-term Service Level Agreement (SLA) between the provider and its customers. The change in demand can therefore be measured with a turnover rate, such as $20 \%$ of incoming (new) and 30\% of leaving (drop or ending) requests. Expressed more precisely, let $P$ be the set of mapping planning periods of time and $M(0)$ the initial set of MEC requests. The set of MEC requests $M(p)$ indexed by $p \geq 1$ is defined as:

$$
M(p)=M(p-1)+M_{\mathrm{NEW}}(p)-M_{\mathrm{DROP}}(p)
$$


Where $M(p-1)$ is the set of accepted MEC requests at the end of period $p-1 . M_{\mathrm{NEW}}(p)$ is the set of new incoming and $M_{\mathrm{DROP}}(p)$ is the set of ending MEC requests at the start of period $p$. Where NEW and DROP are randomly selected between 10 and 40\%, giving us a range of cases from slowly fluctuating (10\%) to quickly changing (40\%) of MEC demand.

\section{Mathematical modeling}

To evaluate the merits of the allocation approach of MECDCs resources, we propose a mathematical formulation as follows.

The MEC-DCs infrastructure is represented by a directed graph $G_{m}=\left(H_{m}, E_{m}\right)$, where $H_{m}$ is the set of MEC-DCs locations, and $E_{m}$ is the set of optical fiber links. The network topology is composed of either a Unidirectional Path Switching Ring (UPSR), consisting of one optical directional fiber, or a Bidirectional Path Switching Ring (BLSR), consisting of two fibers, one in each direction.

End-to-end delay between two MEC-DC nodes is also impacted by the number of MSPP transponders used in the light-path. Figure 1 shows an example of MEC-DCs, where each physical optical link $e \in E_{m}$ between two MEC-DC locations offers $W$ wavelengths, each of which has a bandwidth capacity $B$. We define a light-path $l$ as a set of consecutive optical links. We denote by $L$ the set of light-paths available to serve the networking requirements of media cloud requests $M_{n}, n \in N$. Candidate light-paths can be calculated using a K-shortest path algorithm [27] for all couples of MEC-DC locations that are connected using optical WDM links. Either ring or mesh topologies can be used in calculating the paths. The ring topology is evaluated in "Numerical results" section. It is considered because most current metro topologies are ring-based and still favored over mesh topologies because of their inherent simplicity of design and low OPEX.

Client signals can be added and dropped on any MECDC hosting node $u$ using MSPP transponders $N_{\text {MSPP }}(u)$. In addition, each MEC-DC hosting node $u \in H_{m}$ offers a Compute Processing Unit (CPU) capacity $P_{u}$, a Graphic Processing Unit (GPU) capacity $G_{u}$, a memory capacity $M_{u}$, and a storage capacity $S_{u}$. Table 1 shows the generic description of these parameters.

Similarly, a media cloud request is divisible into a set of interdependent atomic tasks modeled as a weighted, undirected Task Dependencies Graph (TDG) $M_{n}=\left(T_{n}, A_{n}\right)$, where $n \in N=\{1,2, \ldots,|N|\}$. $T_{n}$ denotes the set of Tasks and $A_{n}$ the set of directional virtual networking links between tasks that form media cloud request $M_{n}$. Figure 2 shows an example of a TDG graph. A media cloud request could be, for example, a MapReduce request [32] where the input is a huge amount of data split into smaller parts. The Mapper code is executed on every part, and
Table 1 Notation of MEC-DCs infrastructure

\begin{tabular}{ll}
\hline Parameters & Description \\
\hline$H_{m}$ & Substrate network $G_{m}\left(H_{m}, E_{m}\right)$ \\
$E_{m}$ & Set of substrate nodes \\
$L$ & Set of substrate bidirectional links \\
$W$ & Set of optical light-paths in susbtrate \\
$B$ & Number of wavelength per link \\
$N_{\text {MSPP }}(u)$ & Bandwidth wavelength capacity \\
$P_{u}$ & Number MSPP transponders at node $u$ \\
$G_{u}$ & CPU capacity of node $u$ \\
$M_{u}$ & GPU capacity of node $u$ \\
$S_{u}$ & Memory capacity of node $u$ \\
$C_{u}^{s}$ & Storage capacity of node $u$ \\
$C_{u}^{p}$ & Storage unit price of substrate node $u$ \\
$C_{u}^{m}$ & CPU unit price of substrate node $u$ \\
$C_{u}^{g}$ & Memory unit price of substrate node $u$ \\
$C_{e}^{b}$ & GPU unit price of substrate node $u$ \\
\hline & Bandwidth unit price of substrate link $e$ \\
\end{tabular}

all the results after Sort/Shuffle are sent to one or more reducers that merge all the results into one. More specifically, according to the dependency graph, task T1 is the Splitter/initializer, T2, T3 and T4 are the Mapper, T5 is the Shuffle/Sorter and T6 is the Reducer that combines the results.

Each task $t \in T_{n}$ has a set of VM cloud computing requirements: (a) CPU capacity $p_{t}$, (b) GPU capacity $g_{t}$, (c) memory processing requirement $m_{t}$, (d) storage capacity $s_{t}$, and (e) processing order $o_{t}$ with respect to any $t \in T_{n}$. Similarly, each link $a \in A_{n}$ has networking requirements: (a) data transfer capacity $b_{t t^{\prime}}$ between media service sub-tasks $t$ and $t^{\prime}$, and (b) maximum number of Optical-Electrical-Optical (OEO) conversions $h_{t t^{\prime}}$ of an optical path that links tasks $t$ and $t^{\prime}$, as the time delay in the transport signal is affected mainly by the number of conversions between electrical and optical domains. It should also be noted that loading client signals requires an OEO conversion of the transport signal (wavelength). Table 2 shows the description of these parameters.

Each media cloud request $M_{n}$ can be divided into hosting and network mapping. Each virtual hosting node $t \in$ $T_{n}$ from a media cloud request $n$ is mapped into substrate hosting nodes $u \in H_{m}$ by mapping $M_{\mathrm{N}}: T_{n} \mapsto H_{m}$.

Similarly, each virtual link $a \in A_{n}$ belonging to a media cloud request $n$ is mapped to an optical light-path $l \in$ $l_{u, v}^{a} \subset L$ by mapping $M_{\mathrm{L}}: A_{n} \mapsto L$, where $(u, v)$ are MEC$\mathrm{DC}$ nodes assigned to virtual nodes, respectively the $(s, d)$ source and destination nodes of virtual link $a$.

When a media cloud request arrives, the $C P$ has to determine whether to accept or reject it. This decision 


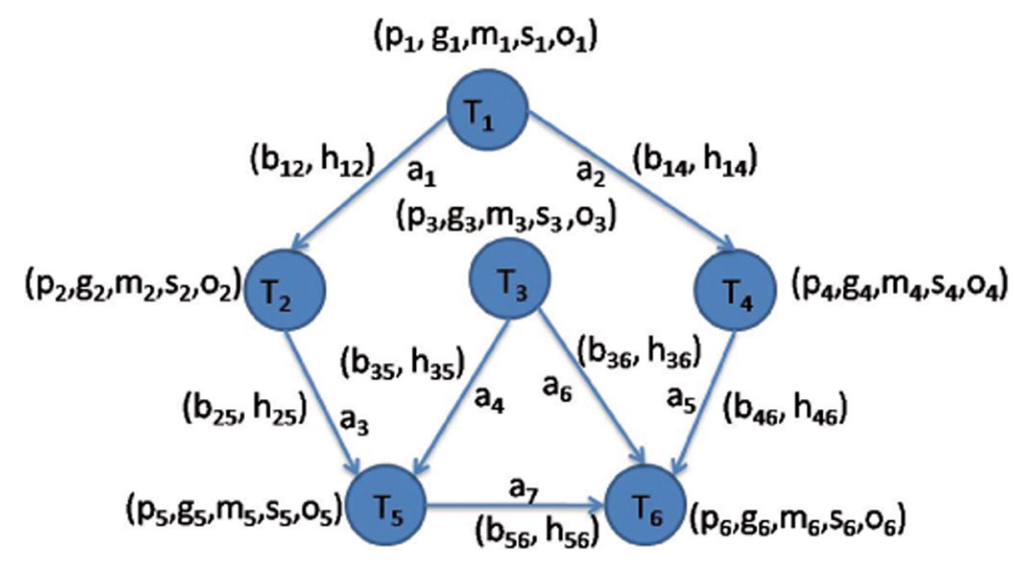

Fig. 2 Task dependencies graph

is largely based on the QoS requirements of the request, the availability of MEC-DCs resources, and the economic cost of accepting the request. Since we are focusing on cloud computing and optical networking, we propose to calculate the mapping cost of each service request $n, G_{n}=\left(T_{n}, A_{n}\right)$, as follows.

$$
\operatorname{COST}\left[M_{n}\right]=\operatorname{COST}\left[M_{\mathrm{N}}\left(T_{n}\right), M_{\mathrm{L}}\left(A_{n}\right)\right] .
$$

Details of mapping cost calculation are provided in the following section.

\section{Column Generation formulation for MEC-DCs resource allocation (L-CG-MEC)}

To avoid the scalability issue identified in the Integer Linear programming formulation [28], we propose the

Table 2 Notation for media cloud requests

\begin{tabular}{ll}
\hline ParametersDescription \\
\hline & Virtual Network $M_{n}\left(T_{n}, A_{n}\right)$ \\
$N$ & Set of virtual network requests \\
$T_{n}$ & Set of tasks in request $n$ \\
$A_{n}$ & Set of directional links between tasks \\
$s_{\text {and } d}$ & Source and destination nodes \\
$p_{t}$ & Required CPU capacity of task $t$ \\
$g_{t}$ & Required GPU capacity of task $t$ \\
$m_{t}$ & Required memory capacity of task $t$ \\
$s_{t}$ & Required storage capacity of task $t$ \\
$O_{t}$ & Processing order of task $t$ \\
$b_{t t^{\prime}}$ & Transfer bandwidth between tasks $t$ and $t^{\prime}$ \\
$h_{t t^{\prime}}$ & Maximum number optical-electrical-optical conversions of a \\
& light-path that links tasks $t$ and $t^{\prime}$ \\
$l_{u, v}^{a}$ & Set of optical light-paths between substrate nodes $u$ and $v$ \\
& used for mapping of virtual link $a$. \\
\hline
\end{tabular}

approach L-CG-MEC using the Column Generation technique [29]. We reformulate the resource allocation problem in terms of Independent Media Cloud Configurations (IMCCs). An IMCC configuration (Fig. 3) defines the mapping solution of at least one MEC request; it is represented by the set of substrate nodes used to handle resource requirements (CPU, memory, GPU and storage) and the links/light-paths, all with the same wavelength, used to connect these nodes. We denote by $C$ the set of all possible IMCCs. The resource allocation problem can then be formulated with respect to the variables $\left(\lambda_{c}\right), c \in C$. Here, variable $\lambda_{c}=1$ if IMCC $c$ is used in the mapping solution and 0 otherwise. In the new formulation, the MEC mapping problem is to choose a maximum of $W$ IMCCs, as $W$ wavelengths are available in each optical fiber link. Each IMCC is mapped on one WDM wavelength. The resulting configuration corresponds to what is known as the master problem in a column generation approach [29], while each IMCC configuration corresponds to what is known as the pricing problem.

An IMCC configuration $c \in C$ is defined by the vector $\left(a_{n}^{c}\right)_{n \in N}$ such that: $a_{n}^{c}=1$ if IMCC $c$ serves media cloud request $M_{n}$ and 0 otherwise. We denote by $\operatorname{CosT}_{c}$ the cost of configuration $c$. This corresponds to the costs of the resources used (hosting and networking) for the set of MEC requests granted by IMCC $c$.

The use of Column Generation divides the original problem into a master problem and a pricing problem: (1) The problem of finding the best subset among the already generated IMCCs that minimize the objective function: mapping resources cost, and (2) the problem of generating an additional column (IMCC) to the constraint matrix of the master problem. 


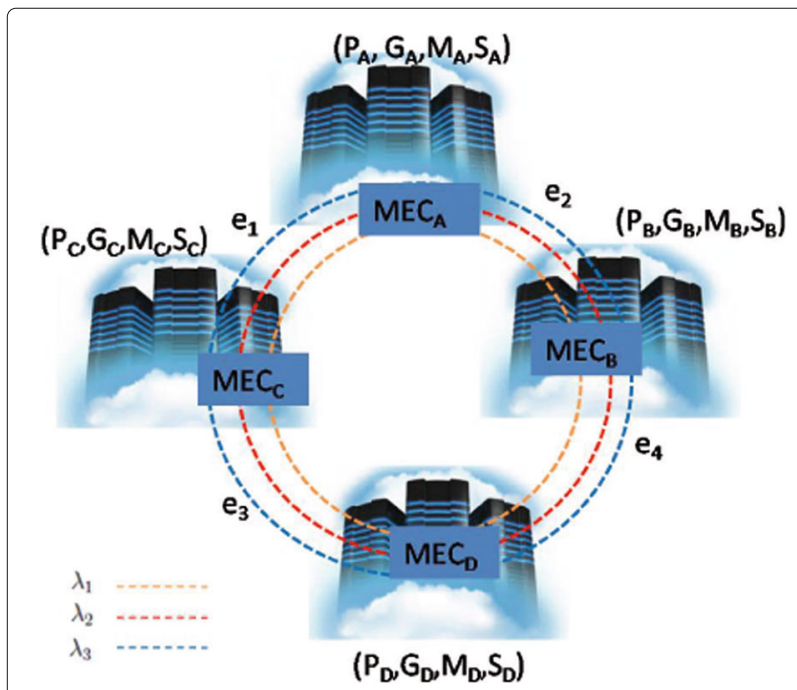

Fig. 3 Combining IMCCs in order to build a mapping solution for media cloud requests

\section{Master problem}

The master problem, denoted by IMCC-ILP, is defined as follows:

\section{Objective function:}

$$
\min \sum_{c \in C} \operatorname{cosT}_{c} \lambda_{c}
$$

where

$$
\begin{aligned}
\operatorname{COST}_{c}= & \sum_{u \in H_{m}} T^{c}(u) \times c_{\mathrm{MSPP}}+\sum_{e \in E_{m}} B^{c}(e) \times c_{e}^{b} \\
& +\sum_{u \in H_{m}} S^{c}(u) \times c_{u}^{s}+P^{c}(u) \times c_{u}^{p}+M^{c}(u) \times c_{u}^{m} \\
& +G^{c}(u) \times c_{u}^{g}
\end{aligned}
$$

$c_{u}^{s}, c_{u}^{p}, c_{u}^{m}, c_{u}^{g}$ and $c_{e}^{b}$ are respectively unit costs of storage, CPU, memory, GPU and bandwidth at MEC-DC location $u$ and optical link $e . T^{c}(u)$ is the number of MSPP transponders used in node $u$ by IMCC $c . c_{\text {MSPP }}$ denotes the unit cost of a MSPP transponder. $B^{c}(e)$ is the bandwidth used on optical link $e$ by IMCC $c$. We also denote by $S^{c}(u), P^{c}(u), M^{c}(u)$ and $G^{c}(u)$ respectively the storage, CPU, memory and GPU in MEC-DC location $u$ used by IMCC $c$. We note that the objective function Eq. (3) minimizes the mapping cost of accepted MEC requests.

\section{Constraints}

$$
\sum_{c \in C} \lambda_{c} \times S^{c}(u) \leq S_{u} ; \quad u \in H_{m} \quad\left(\alpha_{u}\right)
$$

$$
\begin{aligned}
& \sum_{c \in C} \lambda_{c} \times P^{c}(u) \leq P_{u} ; \quad u \in H_{m} \\
& \sum_{c \in C} \lambda_{c} \times G^{c}(u) \leq G_{u} ; \quad u \in H_{m} \\
& \sum_{c \in C} \lambda_{c} \times M^{c}(u) \leq M_{u} ; \quad u \in H_{m} \\
& \sum_{c \in C} \lambda_{c} \leq W ; \\
& \sum_{c \in C} \lambda_{c} \times T^{c}(u) \leq \gamma_{\mathrm{MSPP}}(u) ; u \in H_{m} \\
& \sum_{c \in C} \lambda_{c} \times a_{c}^{n} \geq 1 ; \quad n \in N
\end{aligned}
$$

Equations (5), (6), (7) and (8) guarantee the respect of available physical storage, CPU, GPU and memory capacity respectively. Equation (9) defines the number of WDM wavelengths available per optical link (fiber) $l$ to guarantee the transport of data flows among MEC locations. Equation (10) defines the number of MSPP transponders available in optical network node $u$ to add/drop/groom request flows to/from/with the wavelength available in the optical fibers connecting MEC-DC locations. Equation (11) guarantees that service requests can be satisfied with the available MEC-DCs resources.

\section{Pricing problem}

As mentioned previously, the pricing problem is to generate an additional configuration (IMCC), an additional column, for the constraint matrix of the current master problem. It is defined as follows.

Let $\alpha_{u}, \beta_{u}, \gamma_{u}, \eta_{u}, u_{0}, \zeta_{u}, \theta_{u}$ and $\psi_{n}$ be the dual variables associated with constraints (5), (6), (7), (8), (9), (10) and (11) respectively. Then, the reduced cost of variable $\lambda_{c}$ can be written:

$$
\begin{aligned}
\overline{\operatorname{COST}}_{c}= & \operatorname{COST}_{c}+\sum_{u \in H_{m}}\left(\alpha_{u} \times S^{c}(u)+\beta_{u} \times P^{c}(u)\right. \\
& \left.+\gamma_{u} \times G^{c}(u)+\eta_{u} \times M^{c}(u)+\zeta_{u} \times T^{c}(u)\right)+u_{0} \\
& -\sum_{n \in N} a_{c}^{n} \times \psi_{n}
\end{aligned}
$$

We now express (12) in terms of the decision variables of the pricing problem. Those variables are defined as follows.

- $z_{n}=1$ if media cloud request $M_{n}$ is served by IMCC $c$ and 0 otherwise.

- $y_{u}=1$ if a MSPP transponder is installed in MEC-DC location $u \in H_{m}$ and 0 otherwise. 
- $x_{l}^{a}=1$ if virtual link $a \in A_{n}$ is assigned to light-path $l \in L_{a}$ and 0 otherwise, where $L_{a}$ is the set of light-paths where their lengths do not exceed the number of OEO conversion $h_{t t^{\prime}}$ allowed for a virtual $\operatorname{link} a=\left(t t^{\prime}\right) \in A_{n}, n \in N$.

- $x_{u}^{t}=1$ if task $t \in T_{n}$ is assigned to MEC-DC location $u \in H_{m}$ and 0 otherwise.

Next, we derive the relations between the pricing variables and the coefficients of the master problem. For each $c \in C$ and $n \in N, a_{c}^{n}=z_{n}$, and for each node $u \in H_{m}$, we have:

$$
\begin{aligned}
& S^{c}(u)=\sum_{n \in N} \sum_{t \in T_{n}} s_{t} \times x_{u}^{t} \\
& P^{c}(u)=\sum_{n \in N} \sum_{t \in T_{n}} p_{t} \times x_{u}^{t} \\
& G^{c}(u)=\sum_{n \in N} \sum_{t \in T_{n}} g_{t} \times x_{u}^{t} \\
& M^{c}(u)=\sum_{n \in N} \sum_{t \in T_{n}} m_{t} \times x_{u}^{t} \\
& T^{c}(u)=2 * y_{u}
\end{aligned}
$$

\section{Constraints:}

\section{Mapping of media cloud service tasks}

i. Mapping is done for all tasks of an accepted media cloud request $M_{n}$.

$$
z_{n} \leq \sum_{\left(u, u^{\prime}\right) \in H_{m}^{2}} x_{u}^{t} x_{u^{\prime}}^{t^{\prime}} ;\left(t t^{\prime}\right)=a \in A_{n}, n \in N
$$

ii. A task $t$ of an accepted request $M_{n}$ is assigned to only one MEC-DC location node $u$.

$$
\sum_{u \in H_{m}} x_{u}^{t} \leq z_{n} ; t \in T_{n}, n \in N
$$

\section{Mapping of media cloud request link}

$$
x_{u}^{t} x_{u^{\prime}}^{t^{\prime}} \leq \sum_{l=\left(u, u^{\prime}\right) \in L_{u, u^{\prime}}^{a}} x_{l}^{a} ;\left(u, u^{\prime}\right) \in H_{m}^{2},\left(t t^{\prime}\right)=a \in A_{n} .
$$

Where $L_{u, u^{\prime}}^{a}$ is the set of light-paths between node $u$ and $u^{\prime}$ having a number of O-E-O conversions less than allowed value $h_{t t^{\prime}}$ for virtual link $a=\left(t t^{\prime}\right)$. The consideration of $h_{t t^{\prime}}$ has the following two major roles in determining the optimal solution:

1. It ensures that the number of $\mathrm{O}-\mathrm{E}-\mathrm{O}$ conversions are not more than the maximum allowed for each virtual link of any media cloud request. This is important because metro networks often are not composed of optical amplifiers. The amplifiers are used mostly in Wide Area Networks, with a significant distance between source and destination.

2. It also ensures that data is placed in the closest MEC-DC, which can ensure QoS and cost requirements.

Accordingly, if request $M_{n}$ is accepted, then at least one light-path $l$ is assigned to allow data transfer between tasks $t$ and $t^{\prime}$ assigned respectively to MEC-DC locations $u$ and $u^{\prime}$.

\section{Number of MSPP transponders}

$$
\sum_{n \in N} \sum_{t \in T_{n}} x_{u}^{t} \leq M \times y_{u} ; u \in H_{m}
$$

An add/drop/grooming MSPP transponder port is set up in a MEC-DC location node $u$ if at least one constituent task of a media cloud request is assigned to this location. We note that $M$ is constant and should be equal to or greater than the maximum number of virtual nodes $a \in A_{n}$ that can be mapped to substrate node $u$. $M=G \times N_{\mathrm{MSPP}}(u)$, where $N_{\mathrm{MSPP}}(u)$ is the number of available MSPP transponder in node $u$ and $G$ is the grooming factor, i.e., the number of client signals that can be uploaded on each wavelength using a MSPP transponder.

\section{Wavelength bandwidth capacity}

$$
\sum_{n \in N} \sum_{a \in A_{n}} \sum_{l \in L_{a}} x_{l}^{a} \times \delta_{l}^{e} \times b_{a} \leq B ; e \in L_{m} .
$$

where $b_{a}$ is the bandwidth transfer requirement between any pair of tasks $\left(t, t^{\prime}\right)=a \in A_{n} . B$ is the bandwidth capacity of the wavelength supporting all light-paths and $\delta_{l}^{e}=1$ if light-path $l$ uses optical substrate link $e$.

\section{Wavelength grooming factor}

$$
\sum_{n \in N} z_{n} \leq G
$$

$G$ is the wavelength grooming factor that allows control of the number of media cloud requests that can be loaded on a given wavelength (IMCC). By so doing, CP controls the load and congestion of optical network links. It allows the Cloud Provider to define a maximum use on the most congested links. In addition, it avoids an optical link becoming overloaded, thereby improving the latency experienced.

Linearization of quadratic terms Constraints (18) and (20) include quadratic terms $x_{u^{t}}^{t} x_{u^{\prime}}^{\prime}$. However, since this quadratic term is the product of two binary variables, it can be linearized easily by replacing the this term with 
a new binary variable $y_{u, u^{\prime}}^{t, t^{\prime}}$ where $y_{u, u^{\prime}}^{t, t^{\prime}}=x_{u^{\prime}}^{t} x_{u^{\prime}}^{t^{\prime}}$ and by adding the following constraints.

$$
\begin{aligned}
y_{u, u^{\prime}}^{t, t^{\prime}} & \geq x_{u}^{t} \\
y_{u, u^{\prime}}^{t, t^{\prime}} & \geq x_{u^{\prime}}^{t^{\prime}}
\end{aligned}
$$

Inequalities (24) and (25) ensure that $y_{u, u^{\prime}}^{t, t^{\prime}}$ is zero if either $x_{u}^{t}$ or $x_{u^{\prime}}^{t^{\prime}}$ are zero.

$$
y_{u, u^{\prime}}^{t, t^{\prime}} \geq x_{u^{\prime}}^{t^{\prime}}+x_{u}^{t}-1
$$

Inequality (26) ensures that $y_{u, u^{\prime}}^{t, t^{\prime}}$ takes value 1 if both binary variables $x_{u}^{t}$ or $x_{u^{\prime}}^{t^{\prime}}$ are set to 1 . In our simulation, this linearization technique is done implicitly by the linear solver CPLEX [30].

\section{Solving L-CG-MEC model:}

This section discusses the steps involved in solving the L-CG-MEC model formulated in "Media edge cloud resource allocation approach" section.

\section{Solving linear relaxation of the problem}

We use the following Column Generation method to generate an embedding solution for media cloud requests.

\section{ColumnGenerationProcedure():}

1. Denote LP(M) the continuous relaxation of the master problem ILP(M) obtained by exchanging the integrality constraint (12) by $\lambda_{c} \in[0,1]$ for any $c \in C$.

2. Initialize $\mathrm{LP}(\mathrm{M})$ by a dummy subset, that is, a set of artificial IMCCs with a zero cost.

3. Solve the linear relaxation $\mathrm{LP}(\mathrm{M})$ of the master problem optimally using the CPLEX solver. Then go to step 4.

4. Solve optimally the pricing problem as follows:

(a) First, solve the pricing problem using a heuristic developed based on K-shortest path and the technique of node/link stress function proposed in [14].

(b) If this heuristic generates a new column with a negative reduced cost, go to step 5 .

(c) Otherwise, Solve exactly the pricing problem using the CPLEX solver. Then go to Step 5 .

5. If a column with a negative reduced cost has been found, add this column to the current master problem and repeat Steps 3 and 4. Otherwise, the master problem is optimally solved.

The optimal solution of $\mathrm{LP}(\mathrm{M})$ only provides a lower bound on the optimal integer solution ILP(M). To derive an integer $\mathrm{VN}$ embedding solution, we use the following approach.

\section{L-CG-MEC-B\&B approach}

- Remove relaxation on variable $\lambda_{c}$.

- Apply the classic Branch-and-Bound CPLEX procedure on the optimal solution of the linear relaxation $\mathrm{LP}(\mathrm{M})$ generated using ColumnGenerationProcedure().

\section{Complexity analysis}

How often Cplex solver is used?

The use of the Column Generation technique means that the MEC mapping problem is divided [29] into a master problem (which includes constraints related to the availability of substrate resources) and a pricing problem (which includes the constraints related to the embedding resources used for granted MEC requests). The problem becomes one of generating an IMCC that improves the current value of the master objective function. To check the optimality of a solution of the LP(M) master model, a sub-problem called the pricing problem is solved to try to identify new columns (IMCC configurations) with negative reduced cost.

At each new iteration of the column generation process, the master problem is solved to optimality using a CPLEX solver to guarantee the optimality of the solution obtained at the previous iteration [29]. A CPLEX solver is used to solve the pricing problem only if the heuristic approach based on k-shorted path is unable to find a new IMCC configuration (column) with a negative reduced cost. Accordingly, CPLEX is used only very infrequently to solve the pricing problem at the last iterations of the Column Generation process and to prove the optimality of the mapping solution. By so doing, we speed up the column generation approach and we guarantee the optimality of the $\mathrm{LP}(\mathrm{M})$ solution.

\section{Would determining all IMCCs in an online manner incur significant latency?}

The Column Generation approach addresses the high computation time of the MILP problem by dividing the MEC embedding problem into a set of sub-problems. A sub-problem involves embedding a small number of MEC requests. The solution is represented by an IMCC configuration. Enumerating all IMCCs takes a huge amount of time: there are simply too many, an exponential number. The key concept of the Column Generation optimization approach is that there is no need to enumerate all IMCC configurations. Only a few of them are used to serve the MEC requests and the sub-problems track and generate them. The solving of linear relaxation $\mathrm{LP}(\mathrm{M})$ of the master problem chooses a maximum of $\mathrm{N}$ IMCCs each time to serve the $\mathrm{N} \mathrm{MEC}$ requests. The solving of $\mathrm{LP}(\mathrm{M})$ is done in polynomial time [33] since the number of generated columns is quite small. 


\section{Numerical results}

\section{Simulation benchmark}

To better illustrate the efficiency and superior performance of the Column Generation approach L-CGMEC, we compare our proposal to the performance of three well-known virtual network embedding algorithms found in the literature, using a well defined set of metrics.

- Two-phase mapping approach (2-Phase-Mapping) [14], which first pre-selects the mapping of hosting nodes and then maps virtual links.

- Bin packing [11] (BP), where hosting and network requirements are mapped using a Bin per type of media cloud resource. The Bin packing in [11] introduces a method for forming and classifying Bins based on the resources available. Using the information of Bin classification, the incoming requests are mapped accordingly. The pseudo code for mapping of incoming requests using Bin packing is shown in Algorithm 1.

- Greedy node mapping combined with a K-shortest path algorithm for the link mapping phase (Multi-Site) [18]. The main difference between the greedy approach and Bin packing is that we classify Bins based on the resources. With the greedy approach, the model follows some of the well-known queue approaches such as First In First Out. We arrange the incoming requests in ascending order of their cost. Cost is defined by the function described in equation (2). The requests then are mapped to the MEC-DCs infrastructure. The pseudo-code for the resource allocation is shown in Algorithm 2.

Two main metrics differentiate our proposals from the benchmarks. These are (1) the applied MEC request mapping approach, i.e., one-shot vs. two-phase embedding, and (2) small-batch vs. online mapping. To highlight the advantages of the one-shot node and link embedding approach, we compare our proposal to the 2-PhaseMapping embedding approach. To evaluate the performance of small-batch vs. online embedding, we use BP and Multi-Site as benchmarks.

\section{Experiment setup}

To evaluate the efficiency of the L-CG-MEC model, we carried out experiments using IBM CPLEX solver [30]. The experiments were conducted with a physical infrastructure of 10 MEC-DCs interconnected through a ring metro WDM optical network topology [20]. For each media cloud request, virtual nodes, between 2 and 20 in number, are randomly but uniformly distributed. The minimum connectivity degree is fixed to 2 links. QoS requirements are randomly determined
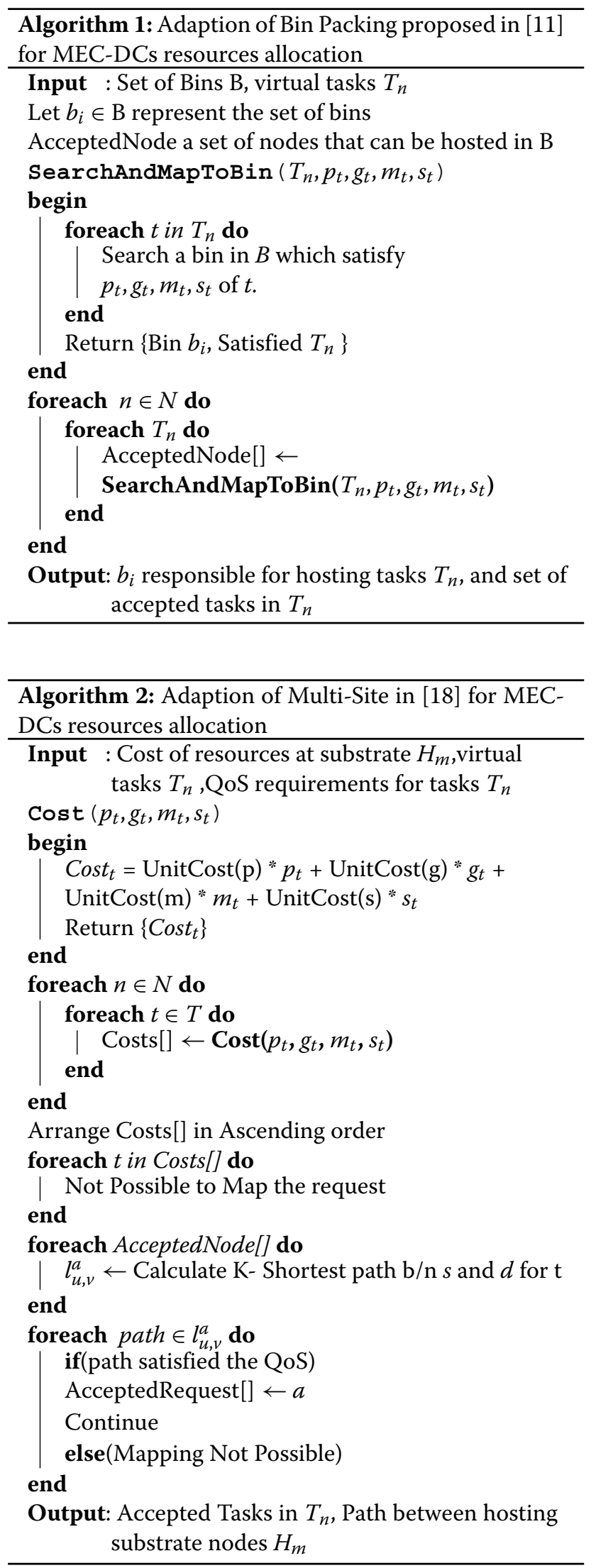
by a uniform distribution among the following QoS classes [31]:

1. High-level delay sensitivity 1 (On-Line Gaming): requires High bandwidth and low latency.

2. High-level delay sensitivity 2 (High Definition Telepresence): requires high bandwidth connection and storage due to high data volume and GPU for real-time audio-visual processing to support a high level of immersion and natural interaction between participants, as in face-to-face meetings.

3. Mid-level delay sensitivity 1 (Live video streaming e.g., sport event): requires minimum latency and high CPU/GPU powers.

4. Mid-level delay sensitivity 2 (Office applications e.g., CRM solutions): requires high storage capacity.

5. Loose-level delay sensitivity (Yahoo and Google Mail): requires loose sensitive delay and high storage capacity.

Bandwidth/CPU/GPU/memory/storage unit cost, are expressed in terms of $\$ X$, which represents the price of 1 $\mathrm{Mb}$ of bandwidth or 1 unit of CPU/GPU/GB.

\section{Performance evaluation metrics}

In our experiments, we evaluated the following metrics.

1. Mapping Cost: The cost of the MEC-DCs resources used.

2. Media cloud demands' blocking ratio: Blocking ratio measures the overall number of rejected MEC requests at each embedding period. It is the ratio of the number of rejected requests to the overall number of requests. While it gives a sense of how well an algorithm is performing, it cannot completely capture the performance and customer satisfaction, as these depend on the quality and the cost of the service. In fact, depending on the cost of MEC requests, it is possible for $10 \%$ of MEC requests, as an example, to provide a revenue equivalent to that offered by the remaining requests.

3. Wavelength utilization: The average ratio between the used and the overall amounts of available wavelength bandwidth.

4. CPU/GPU/Storage utilization: The ratio between the used and the overall available amounts.

5. Average number of hops: The average number of hops per mapped virtual link.

\section{Evaluation results}

This section describes the performance of the L-CG-MEC approach compared to related works in terms of resource usage. We also analyze the key factors that impact the optical transmission network among MEC-DC locations (wavelength grooming factor, number of wavelengths, and the network linking topology (i.e., UPSR vs. BLSR)).

\section{Efficiency of light-path resource allocation approach}

We first study the performance of the proposed L-CGMEC model compared to the benchmarks in terms of mapping cost, media cloud blocking ratio, CPU, GPU storage and wavelength bandwidth usage.

Figure 4a plots the cumulative CP mapping cost against the allocation time periods. It compares the mapping cost for L-CG-MEC and for benchmark models BIN, 2Phase-Mapping and Multi-site. The results show that the L-CG-MEC model provides the lowest cost by approximately $37 \%$ compared to Multi-Site, which had the highest cost during the simulation run period 10 . The cost for the greedy approaches, Multi-Site and BP, is high because they inherently map requests without optimizing. By contrast, the 2-phase-mapping solution is solved for optimization, resulting in minimal cost.

Figure $4 \mathrm{~b}$ plots the blocking ratio of media cloud requests against the allocation time periods. The Bin and Two-Phase-Mapping approaches show a major blocking ratio on some periods and a higher cumulative cost. MultiSite provides the lowest blocking ratio, but at the highest
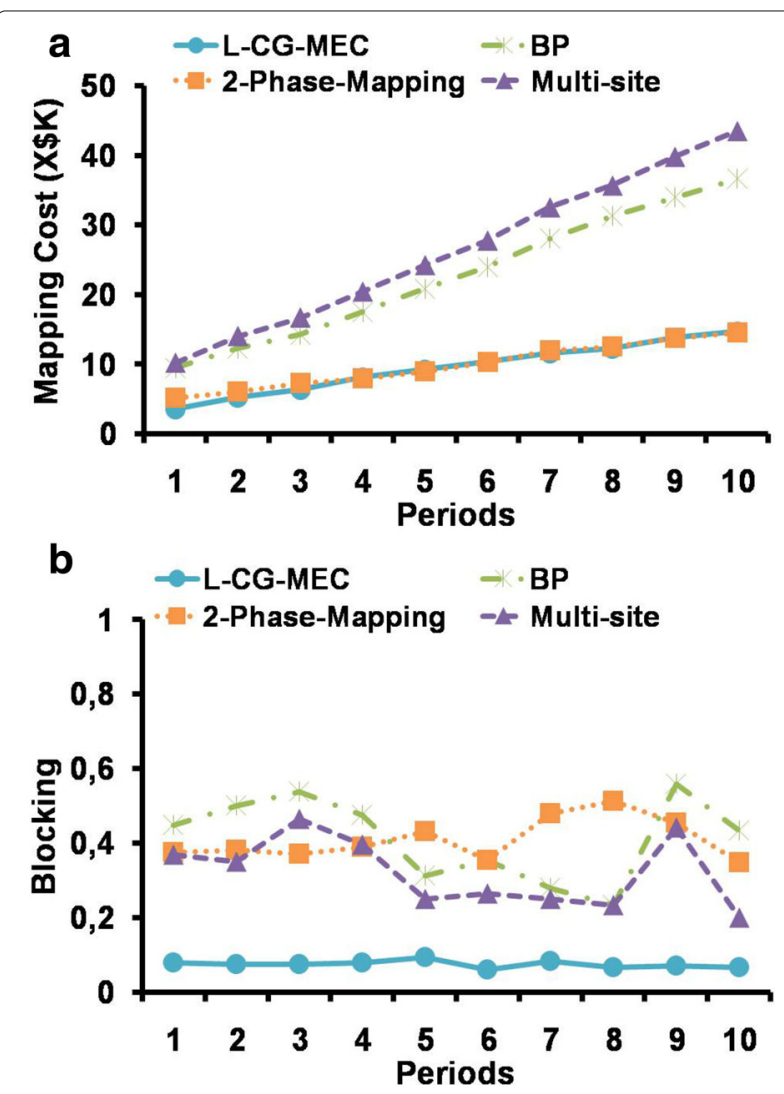

Fig. 4 Performances L-CG-MEC vs. benchmarks. a Mapping cost. b Blocking ratio 
cost. This is because, with the exception of L-CG-MEC, no approach performs global optimization. Therefore, the solution determined at the node phase cannot be satisfied during the light-path mapping phase because resources in the selected paths are not available. On the other hand, with L-CG-MEC, requests are blocked because the incoming request requirements were beyond those the infrastructure service provider could satisfy at that time.

For every period, several combinations of different classes of traffic are generated to mimic the real traffic. This explains the jagged nature of the substrate resource graphs (Fig. 5 and 6). In other words, if, in period one, $10 \%$ of the traffic is class 1 (online gaming traffic) and the remaining $90 \%$ of the traffic is class 2 (mail traffic), the resource requirement is lower and acceptance is higher compared to a scenario where $80 \%$ of the traffic is class 5 and remaining $20 \%$ is class 1 .

Figure $5 \mathrm{a}$ shows the wavelength use of the selected approaches compared to L-CG-MEC. A higher wavelength use in an optical network can be related to higher link throughput. The higher the wavelength use, the better the system. At the same time, care needs to be taken that selected paths for the requests do not perform too many O-E-O conversions (more detail is given in Impact

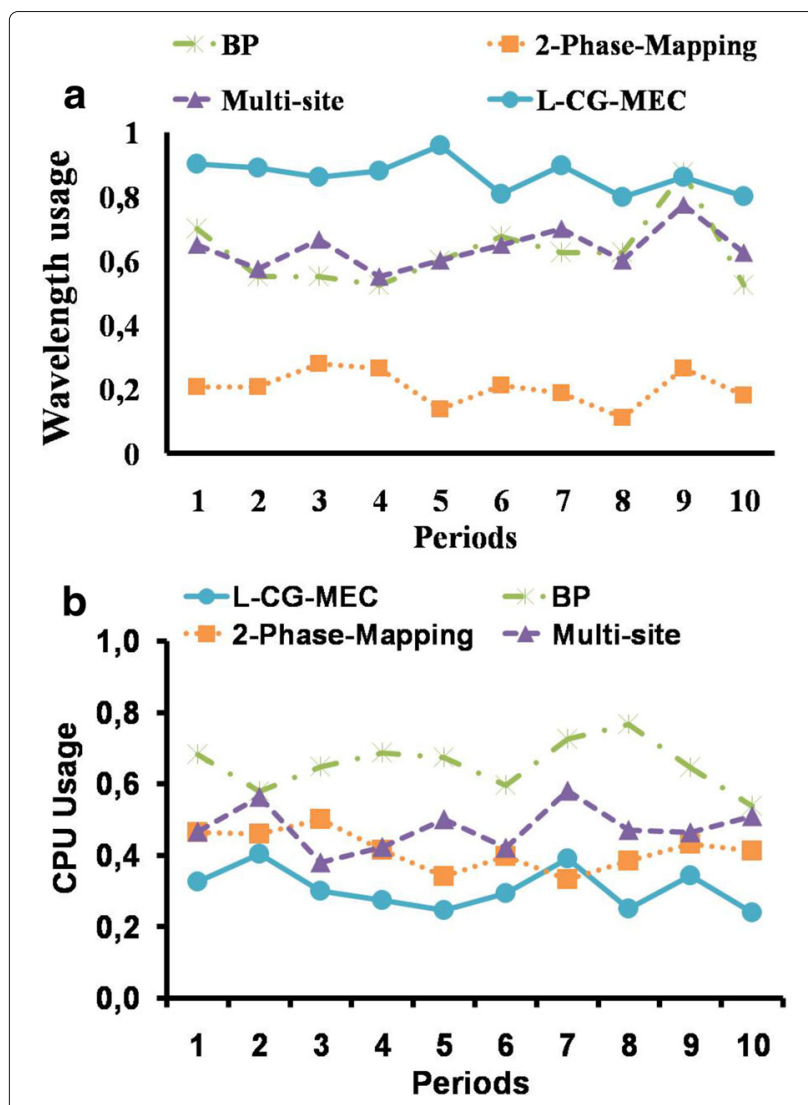

Fig. 5 MEC-DCs Resource utilization for L-CG-MEC vs. Benchmark approaches. a Wavelength bandwidth usage. b CPU usage
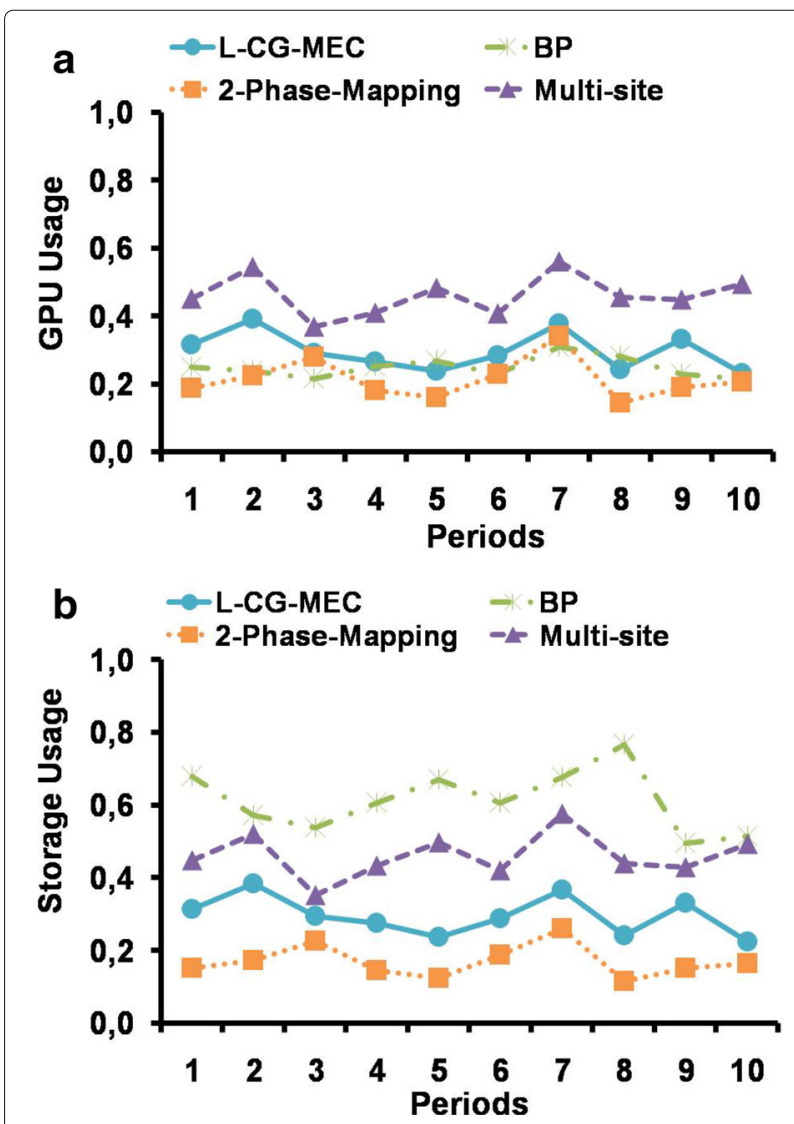

Fig. 6 Periodical MEC-DCs nodes resources usage. a GPU usage. b Storage usage

of number of wavelengths per optical Fiber section). From the results, it is clear that 2-phase mapping has the least bandwidth use. Although 2-phase had better acceptance, the wavelength use was poor. This is due to an improper selection of requests accepted for the embedding. The number of requests could be higher but the revenue generated from the request leads to too much wastage of resources. The L-CG-MEC had the highest overall wavelength use because an initial global optimization was performed. This solution yielded the overall optimal solution in terms of the highest use of link resources at the lowest cost to the service providers. A similar conclusion can be drawn from the other approaches, BP and multi-site, where lack of optimization and a one-shot solution can lead to poor use of link resources. Hence, we can ascertain from Fig. 5a that a global optimization, if designed with proper constraints, provides better resource use and less complexity.

Figures $5 \mathrm{~b}, 6 \mathrm{a}$ and $\mathrm{b}$ show the QoS resource usage in different approaches. Although in Fig. $6 a$ and 6b, 2phase-mapping had better overall use of GPU and storage resources, we can see that L-CG-MEC had better (i.e. least) use of wavelength, CPU, GPU and Storage. The 
worst performance was by BP, because of its first-comefirst-served approach.

\section{Analysis of network dimensioning key factors}

Below, we analyze the key factors that impact MEC-DCs resource utilization and uses QoE i.e., acceptance ratio, number of hops, and mapping cost.

Impact of grooming factor Figures $7 \mathrm{a}, \mathrm{b}$ and $\mathrm{c}$ plot the variation of mapping cost, the average number of hops and the wavelength bandwidth use against the grooming factor (the number of client signals that can be loaded on a given wavelength). The figures show the impact of the grooming factor on the L-CG-MEC model in terms of bandwidth wavelength use and the average number of hops. These two parameters have a big impact on latency, the most stringent QoS requirement. The grooming factor, in fact, needs to be kept as low as possible in order to avoid congestion and to guarantee an acceptable degree of latency in multimedia applications. These figures show grooming factor values that provide the optimal average number of hops per service request link as well as the optimal wavelength use. In other words, the results of the simulation illustrate the adjustment of the grooming factor with respect to expected wavelength bandwidth use and the average number of hops needed to keep the latency below accepted values.
Impact of number of wavelengths per optical Fiber Figures $7 \mathrm{~d}, 8 \mathrm{a}$ and $\mathrm{b}$ plot the variation of blocking ratio, mapping cost and average number of hops against the number of wavelengths used per optical fiber link. First, the blocking ratio is clearly closely related to the number of wavelengths used to transmit service request data among MEC-DC locations. The blocking ratio actually decreases as the number of wavelengths increases. Second, these figures show that, for a given demand pattern, increasing the number of wavelengths beyond a certain value has no impact on mapping cost and the average number of hops. For example, when the number of wavelengths equals 5 , the mapping cost and the average number of hops are quite constant. These results help to optimize capital expenditures while honoring the QoS requirements inherent in media cloud demand. Also, the number of hops is crucial in an optical network as it might lead to O-E-O conversion. Care needs to be taken to reduce O-E-O conversion as the system strives to increase its link resource use.

Impact of linking topology Figures 9a, b and c plot respectively the average number of hops, blocking ratio and mapping cost against the allocation time periods. The results show the impact of the network topology on the performance of the proposed L-CG-MEC approach.
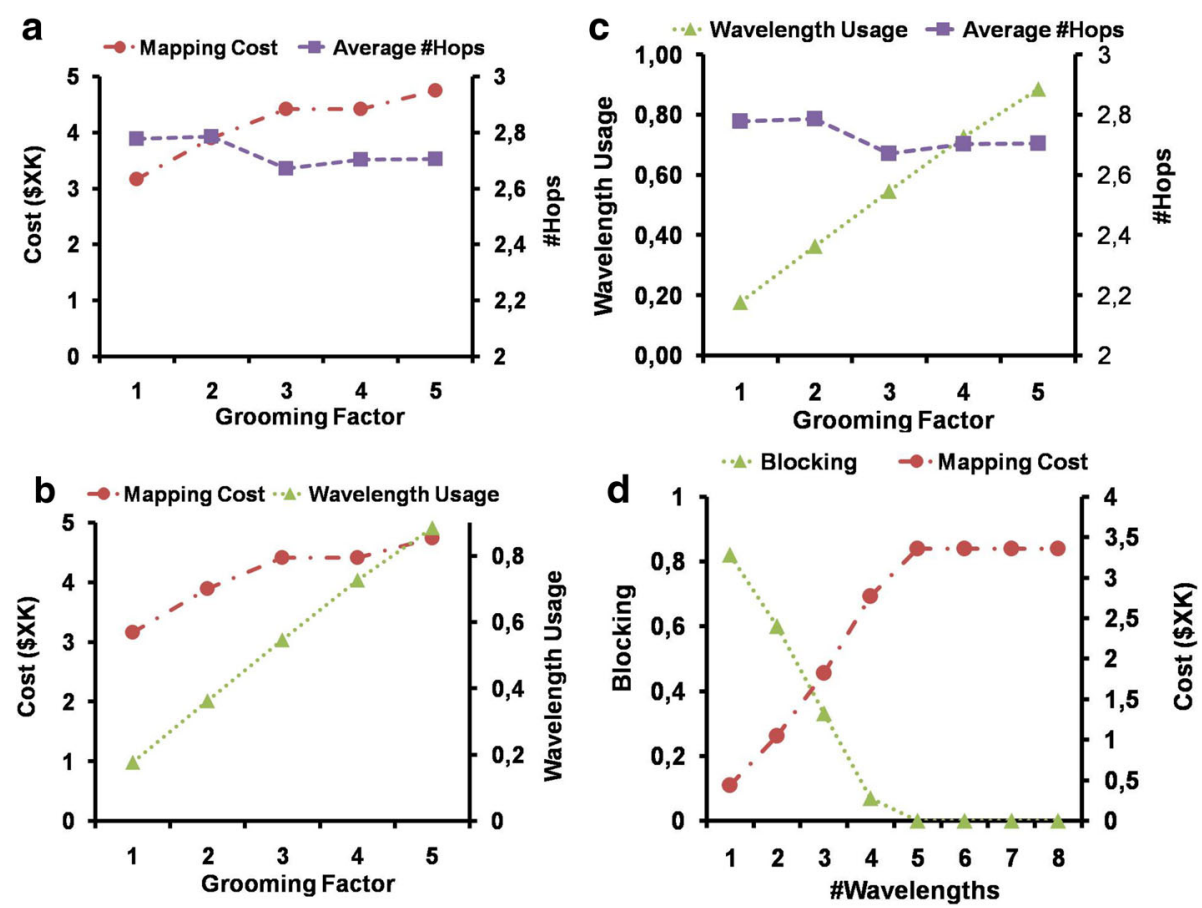

Fig. 7 Impact of Grooming factor. a Mapping cost and Number of hops. b Wavelength use and mapping cost. c Wavelength use and Number of hops. d Blocking and mapping cost 


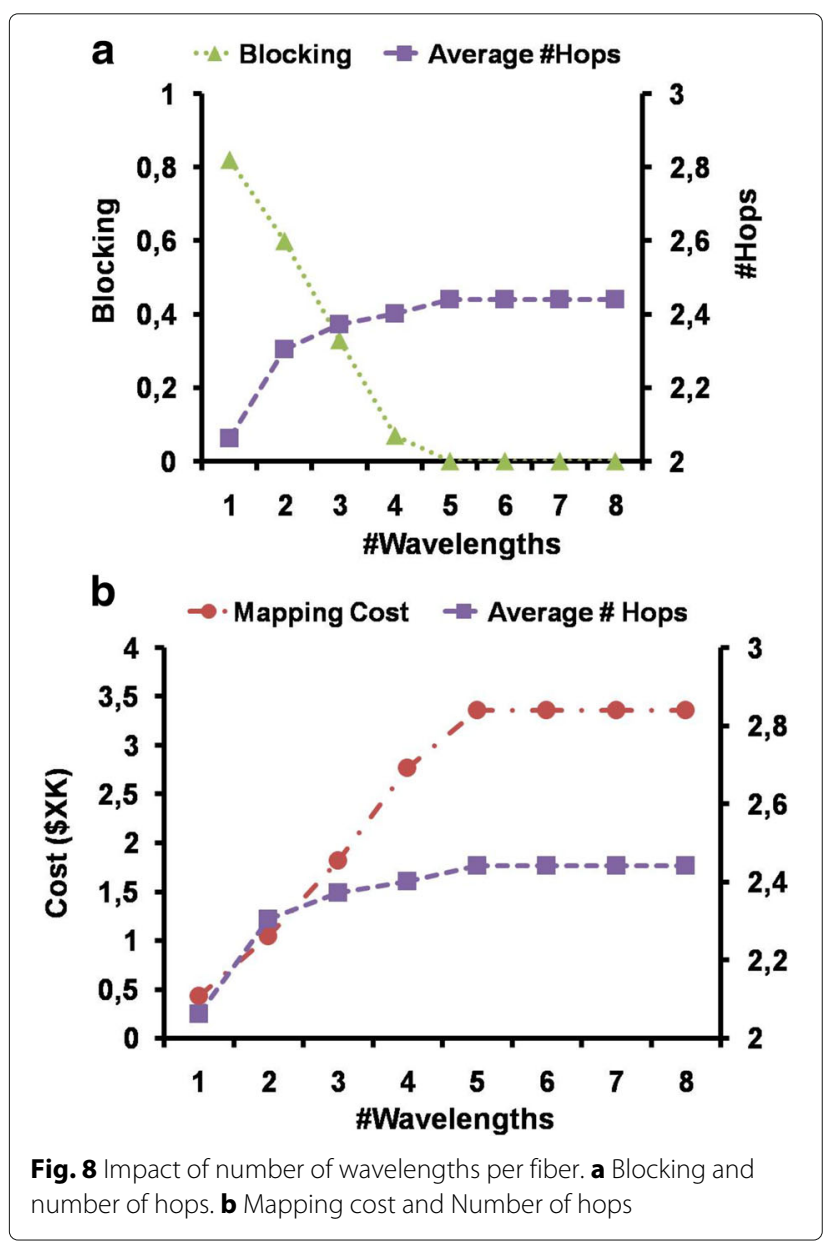

UPSR and BLSR topology are the most widely used configurations in optical networks. We therefore studied mapping performances in comparison to UPSR vs BLSR topologies. The results show that BLSR topology reduces the average number of hops per mapped virtual link. However, both topologies provide almost equal mapping costs and blocking ratios. We can conclude that the use of UPSR and BLSR has a minimal effect on the initial acceptance or performance of the user requests. On the other hand, UPLSR and BLSR have an impact in providing resilience to the accepted requests. Nonetheless, the proposed model can be used with both UPSR and BLSR metro networks.

\section{Simulation CPU time}

Only one network configuration is used in our simulation and this may limit the generalization of the results. It is hard to give a clear relationship between CPU time and network parameters (\# nodes, \# links) and traffic pattern/volume. However, although there might be a significant impact in increasing the network parameters (nodes, links) on simulation CPU time, our Column Generation approach calculates a feasible and near-optimal solution by heuristically solving the pricing problem with a large network and a large number of requests. As for any network design problem, it is expected that the larger the numbers of network nodes and links, the longer the CPU time. There is a certain trade-off between reducing the simulation CPU time and calculating the optimal solution. In addition, the current solution is suggested for metro

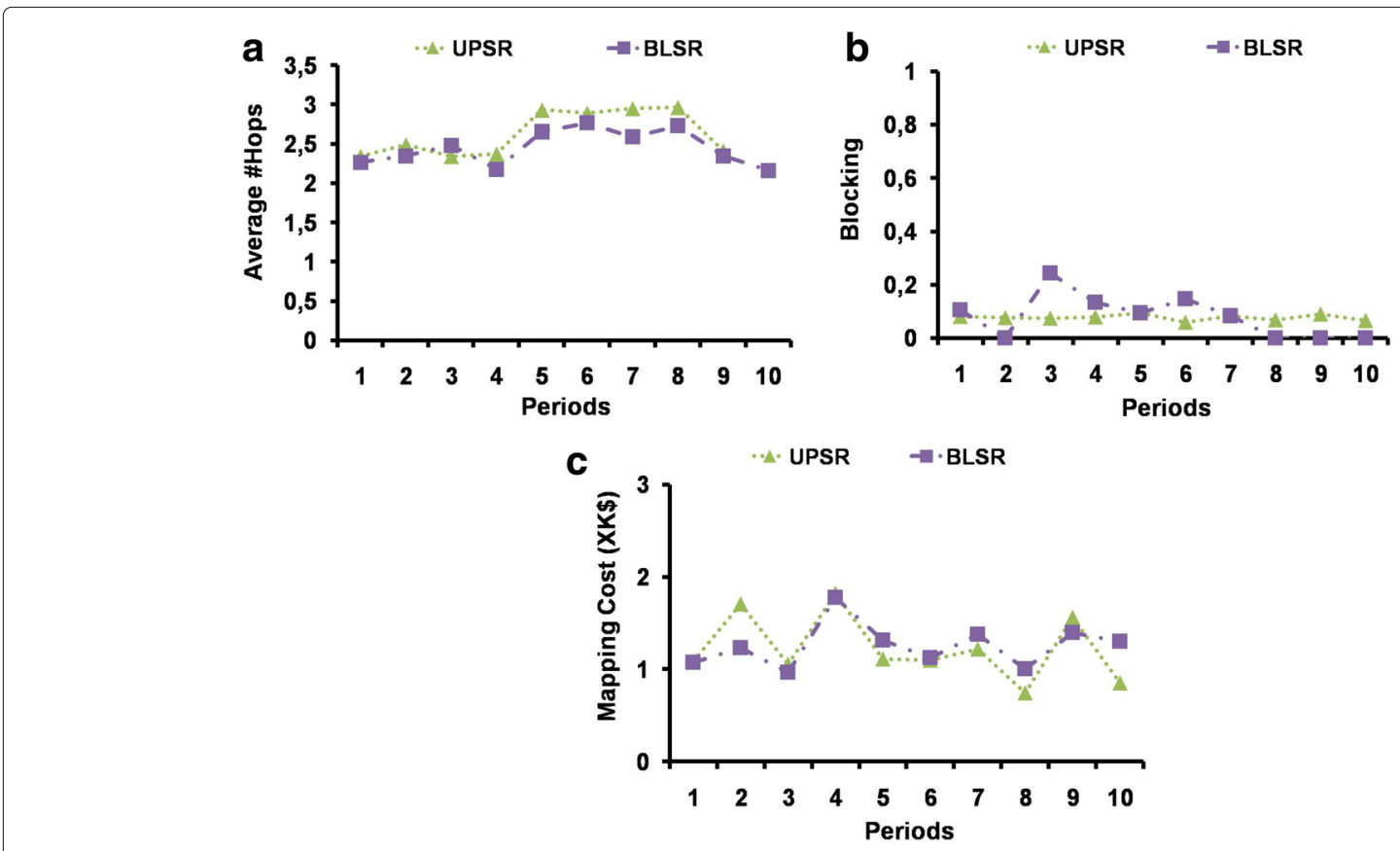

Fig. 9 Performance UPSR vs. BLSR topologies. a Number of Hops. b Blocking ratio. c Mapping cost 
optical ring topology where the number of nodes/links and the number of requests are relatively less important than with meshed and large-scale networks. According to the simulation results, the L-CG-MEC decomposition approach showed a higher computation time than the benchmarks. The two benchmarks showed a computation time of a few seconds to a few minutes and L-CG-MEC a computation time varying from 10 to 20 minutes. However, we showed that Column Generation L-CG-MEC decreased the MEC mapping cost by $37 \%$ and blocking ratio by $15 \%$ on average and that MEC resources are used more efficiently.

One might ask whether the significant difference in computation time between the benchmarks and L-CGMEC is reasonable. Yes, it is. The benchmarks use a heuristic approach, which means using a local search algorithm to browse for feasible solutions. Only a small number of possible solutions are examined, so computation time is short. But the heuristic approach is unable to indicate how far the solution obtained is from the optimal one. However, L-CG-MEC is based on an exact approach (ILP modelling) using a global search algorithm to browse for feasible solutions. Accordingly: (a) the computation time increases significantly with the size of the space, and (b) an exact algorithm can consume a lot of memory, also leading to a high computation time. Nevertheless, the L-CG-MEC approach provides the gap between the obtained solution and the optimal one, even when the algorithm is stopped before completion. It can even prove optimality, if the integer embedding solution is equal to the optimal lower bound provided by the linear relaxation of the master problem $\mathrm{LP}(\mathrm{M})$.

Computation times are obtained using a CPLEX solver 12.3 and an Intel Duo Core Dell machine running Windows 7 Enterprise. Since L-CG-MEC uses a Branch-andBound algorithm to find an integer solution, we cannot claim that we have calculated the optimal solutions. However, the solutions are still satisfactory compared to those obtained using benchmark models. In addition, the difference between the value of the incumbent integer solution of $\operatorname{ILP}(M)$ model and the optimal value of the linear relaxation $L P(M)$ is smaller than $5 \%$. This is satisfactory, given that most proposals in the literature are heuristic or based on two-phase mapping and that one-shot node and link embedding is a NP-hard problem. For an optimal solution, the L-CG-MEC approach can be combined with branch-and-price procedure, where branching rules can be properly defined to avoid generating a huge number of pricing problems. Branching can be done either on the variables of the master problem using cuts, or on the variables of the pricing problem, using a classic branchand-bound procedure or cuts. We propose to examine this technique in our future work.

\section{Conclusion}

Processing, transmitting and storing media data in MECDCs can enhance the QoE of an end user in terms of latency, acceptance ratio, reliability and cost. The use of MEC-DCs compared to traditional DCs for media requests has known limitations: limited resource availability at a given $\mathrm{DC}$, and a high resource requirement. To efficiently manage both the network and the MEC-DCs resources, we proposed a CG-based one-shot model. For each accepted request, an optimal one-shot networking and hosting scheme is calculated to ensure QoS requirements. We also analyzed the key factors that impact the network among MEC-DC locations: wavelength grooming, wavelength bandwidth capacity, the number of wavelengths per optical link, the number of MSPP transponders and the impact of network topology (UPSR vs. BLSR). Simulation results proved that the L-CG-MEC approach performed significantly better compared to the benchmark approaches from the literature.

As a future improvement, we would like to consider Dense Wavelength Division Multiplexing factors and study their impact on QoE of multimedia application users.

\section{Abbreviations \\ laaS: Infrastructure as a service; CS: Compresssive sensing; CP: Cloud service providers; L-CG-MEC: Large scale column generation media edge cloud; MEC-DC: Media edge cloud data centers; MSPP: Multi service provisioning platform; QoE: Quality of experince; SLA: Service level agreement; SLA: Service level agreement; QoS: Quality of service; UGC: User generated content; VM: Virtual machine; VN: Virtual network; WDM: Wavelength division multiplexing; WMN: Wireless mesh network: \\ Funding \\ This work was supported by a Grant from Natural Sciences and Engineering Research Council of Canada. The funding agency is not involved in this research.}

\section{Availability of data and materials \\ Not applicable.}

\begin{abstract}
Authors' Contributions
This paper describes a collaborative project between the University of Ottawa, Canada, and the University of Paris Descartes, France. AJ: Design, modelling, analysis and interpretation of data, prepare first draft of the paper. AK: Design, revise and give final approval of paper. JS: Implementation, simulation, analysis and interpretation of data, drafted the paper. JE: Design and revise the paper. AM: Design and final approval of the paper. FZ: Draft and revise the paper. All authors have read and approved the final manuscript.
\end{abstract}

Competing interests

The authors declare that they have no competing interests.

\section{Publisher's Note}

Springer Nature remains neutral with regard to jurisdictional claims in published maps and institutional affiliations.

Received: 5 July 2017 Accepted: 22 November 2017

Published online: 12 December 2017 


\section{References}

1. Xu H, Li B (2012) A general and practical datacenter selection framework for cloud services. IEEE 5th International Conference on Cloud Computing (CLOUD), Honolulu, HI, USA. pp. 9-16

2. Fesaehaye D, Gao Y, Nahrstedt K, Wang G (2012) Impact of Cloudlets on interactive mobile cloud application. IEEE 16th Conference International Enterprise Distributed Object Computing (EDOC), Beijing, China. pp 123-132

3. Endo P, et al. (2011) Resource allocation for distributed cloud: concepts and research challenges. IEEE Network 25(4)

4. Zhang Q, Zhani M, Jabri M, Boutaba R (2014) Venice: Reliable virtual data center embedding in clouds. INFOCOM:289-297

5. Hou W, Guo L, Liu Y, Yu C, Zong Y (2015) Resource management and control in converged optical data center networks: survey and enabling technologies. Computer Networks 88:121-135

6. Bari DF, et al. (2013) Data-Center Network Virtualization: A Survey, Communications Surveys and Tutorials 15(2):909-928

7. Pillai P, Lewis G, Simanta S, Clinch S, Davies N, Satyanarayanan M (2013) The Impact of Mobile Multimedia Applications on Data Center Consolidation. IEEE International Conference Cloud Engineering (IC2E), Redwood City, CA,USA. pp 166-176

8. Zhu W, Luo C, Wang J, Li S (2011) Multimedia cloud computing. IEEE Signal Process Mag 28:59-69

9. Peng S, Nejabati R, Simeonidou D (2013) Role of Optical Network Virtualization in Cloud Computing. IEEE/OSA J Opt Commun Netw 5(10):162-170

10. Jarray A, Jaumard B, Houle AC (2010) Reducing the CAPEX and OPEX Costs of Optical Backbone Networks. IEEE International Conference on Communications (ICC), Cape Town, South Africa

11. Eyraud-Dubois L, Larcheveque H (2013) Optimizing Resource allocation while handling SLA violations in Cloud Computing platforms. IEEE 27th International Symposium on Parallel \& Distributed Processing (IPDPS), Boston, MA, USA

12. Zeng $L Z$, Ye X (2012) Multi-objective Optimization Based Virtual Resource Allocation Strategy for Cloud Computing. IEEE/ACIS 11th International Conference on Computer and Information Science (ICIS), Shanghai, China

13. Nonde L, et al. (2014) Green Virtual Network Embedding in Optical OFDM Cloud Networks. 16th International Conference on Transparent Optical Networks (ICTON), Graz, Austria

14. Zhu Y, Ammar MH (2006) Algorithms for Assigning Substrate Network Resources to Virtual Network Components. IEEE Infocom:1-12

15. Chowdhury NMK, Rahman MR, Boutaba R (2012) ViNEYard:Virtual Network Embedding Algorithms With Coordinated Node and Link Mapping. ACM/IEEE Transaction on Networking 20(1):206-2019

16. Thompson K, Miller GJ, Wilder R (1997) Wide-Area Internet Traffic Patterns and Characteristics. IEEE Network 11(6):10-23

17. Jarray A, et al. (2012) DDP: A Dynamic Dimensioning and Partitioning model of Virtual Private Networks resources. Comput Commun 35:906-915

18. Nan $X$, He Y, Guan L (2012) Optimal Resource Allocation for Multimedia Application Providers in Multi-site Cloud. IEEE International Symposium on Circuits and Systems (ISCAS), Beijing, China

19. Tzanakaki A, et al. (2011) Energy Efficiency in integrated IT and Optical Network Infrastructures: The GEYSERS approach. IEEE Conference on Computer Communications Workshops (INFOCOM WKSHPS), Shanghai, China

20. Gokhale P, Kumar R, Das T, Gumaste A (2010) Cloud computing over metropolitan area WDM networks: the light-trails approach. IEEE Global Telecommunications Conference (GLOBECOM 2010), Miami, FL, USA

21. Tzanakaki A, Anastasopoulos MP, Zervas GS, Rofoee BR, Nejabati R, Simeonidou D (2013) Virtualization of heterogeneous wireless-optical network and IT infrastructures in support of cloud and mobile cloud services. IEEE Commun Mag 51 (8):155-161

22. Hou W, Guo L, Liu Y, Song Q, Wei X (2013) Virtual Network Planning for Converged Optical and Data Centers: Ideas and Challenges. IEEE Network 27(6):52-58

23. Anastasopoulos MP, Tzanakaki A, Simeonidou D (2015) Scalable services provisioning in converged optical wireless clouds using compression techniques. OFC
24. Develder C, Buysse J, Dhoedt B, Jaumard B (2014) Joint dimensioning of server and network infrastructure for resilient optical grids/clouds. IEEE/ACM Trans Netw 22(5):1591-1606

25. Gong X, Guo L, Shen G, Tian G (2017) Virtual Network Embedding for Collaborative Edge Computing in Optical-Wireless Networks. in J Lightwave Tech 35(18):3980-3990

26. Hou W, Ning Z, Guo L, Chen Z, Obaidat MS (2017) Novel Framework of Risk-Aware Virtual Network Embedding in Optical Data Center Networks. in IEEE Syst J PP(99): 1-10

27. Eppstein D (1998) Finding the shortest paths. SIAM J Comput 28(2):652-673

28. Anderson DG (2002) Theoretical approaches Approaches to Node Assignment. Unpublished Manuscript, available at: https://www.cs.cmu edu/ dga/papers/andersen-assign-abstract.html. Accessed Dec 2017

29. Ubbecke ME, Desrosiers J (2005) Selected Topics in Column Generation. Operations Res 53:1007-1023

30. IBM ILOG Cplex 12.6.1, User Manual. http://pic.dhe.ibm.com/infocenter/ cosinfoc/v12r4/topic/ilog.odms.studio.help/pdf/usrcplex.pdf. Accessed Dec 2017

31. Ceselli A, Premoli M, Secci S (2015) Cloudlet Network Design Optimization. IFIP

32. Dean J, Ghemawat S (2008) Magazine Communications of the ACM - 50th anniversary issue: 1958 - 2008 51(1):107-113

33. Schrijver A (1986) Theory of Linear and Integer Programming. Wiley, New York

\section{Submit your manuscript to a SpringerOpen ${ }^{\circ}$ journal and benefit from:}

- Convenient online submission

Rigorous peer review

- Open access: articles freely available online

- High visibility within the field

- Retaining the copyright to your article

Submit your next manuscript at $\boldsymbol{~ s p r i n g e r o p e n . c o m ~}$ 